\title{
Contribution à la connaissance de la diversité et endémisme des Rubiaceae du Parc National de Kahuzi-Biega à l'Est de la R.D. Congo
}

\author{
Jean-Claude MWANGA MWANGA ITHE ${ }^{1}$, Gérard IMANI MUGISHO ${ }^{2}$, \\ Pazo WABIKA DUMBO ${ }^{1}$, Félicien MUSHAGALUSA KASALI ${ }^{3}$ et \\ Jean de Dieu MANGAMBU MOKOSO ${ }^{4,5^{*}}$
}

\footnotetext{
${ }^{1}$ Centre de Recherche en Sciences Naturelles (CRSN/Lwiro), Département de Biologie, Laboratoire de Systématique et Taxonomie Végétale, DS Bukavu, R.D. Congo.

${ }^{2}$ Université Officielle de Bukavu, Faculté des Sciences et Sciences Appliquées, Département d'Ecologie et Gestion des Ressources Végétales, Laboratoire d'Ecologie Appliquée et Aménagement Forestier, B.P- 570 Bukavu (R.D. Congo).

${ }^{3}$ Université Officielle de Bukavu, Faculté de Médecine et Pharmacie, Département de Pharmacie, Laboratoire de Pharmacognosie et des Recherche sur les Substances Naturelles, B.P-570 Bukavu, R.D. Congo.

${ }^{4}$ Université Officielle de Bukavu, Faculté des Sciences et Sciences Appliquées, Département d'Ecologie et Gestion des Ressources Végétales, Laboratoire Biogéographie et Systématique des Plantes Vasculaires, B.P-570 Bukavu, R.D. Congo.

${ }^{5}$ Université d'Anvers/Belgique, Département de Biologie, Laboratoire d'Ecologie Végétale et Management des Ecosystèmes, Universiteitsplein 1-C, B-2610 Antwerp-Wilrijk, Belgium.

*Auteur correspondant, E-mail : JeandeDieu.MangambuMokoso@student.ua.ac.be; mangambu2000@yahoo.fr
}

\section{RÉSUMÉ}

Nous présentons dans cet article la diversité et l'endémisme des Rubiaceae collectées au Parc National de Kahuzi-Biega (PNKB) se trouvant dans les herbariums de Lwiro (LWI) et Mulungu (MLGU). Et nous mettons à jour les données taxonomiques et écologiques de cette famille au PNKB dans les trois sites ciblés (zone des montagnes, zone de la plaine à Itebero et Lulingu). La méthode taxonomique d'herbier a été utilisée sur les observations macroscopique et microscopique des spécimens. Les Rubiaceae sont représentées au PNKB par 41 genres, 63 espèces, 13 taxa infraspécifiques et 18 (spécimens) restent à identifier. Le genre Psychotria domine avec 11 espèces, suivi de Rytigynia, Tricalysia. 10 genres et 12 taxa infraspécifiques ont été ajoutés sur la liste floristique de Fischer. La partie montagnarde est plus diversifiée en Rubiaceae que la basse altitude. Trois espèces (Pauridiantha kahuziensis, Psychotria bugoyensis et Sericanthe leonardii. subsp. leonardii) ont été identifiées comme endémiques du PNKB. Les résultats montrent que la connaissance de la flore de la RDC reste encore fragmentaire malgré plus d'un siècle de multiples récoltes végétales.

(C) 2013 International Formulae Group. All rights reserved.

Mots clés : Diversité, endémisme, Rubiaceae, Parc National de Kahuzi-Biega, RD. Congo.

\section{INTRODUCTION}

Situé à cheval sur l'équateur et au centre de l'Afrique subsaharienne, la
République Démocratique du Congo (RDC), avec ses 17 grandes formations végétales, s'étend sur environ $2346201 \mathrm{~km}^{2}$ et regorge 
une faune et une flore variées notamment dans ses réserves naturelles et ses parcs nationaux (Vancutsem et al., 2006). Parmi ces Parcs, figure le Parc National de KahuziBiega (PNKB) situé au carrefour des centres d'endémismes Guinéo-Congolais et Afromontagnard (Plumptre et al., 2007 ; Mangambu et al., 2010). Il s'étend sur 6000 $\mathrm{km}^{2}$ situés entre $1^{\circ} 36^{\prime}-2^{\circ} 37^{\prime}$ latitude Sud et $27^{\circ} 33^{\prime}-28^{\circ} 46^{\prime}$ longitude Est (Yamagiwa et al., 2005). Il fut créé pour la protection des gorilles des plaines orientales, Gorilla beringei graueri Matschie et leur habitat (Yamagiwa et al., 2005 ; Mangambu et al., 2013).

La flore du PNKB a été connue grâce aux multiples missions effectuées par les experts étrangers venus pour de grandes expéditions pendant et après l'époque coloniale. Les matériels récoltés sont conservés à l'Institut National d'Etude et de la Recherche Agronomique/Mulungu (MLGU) et à 1'Institut de Recherche Scientifique en Afrique Centrale, actuellement Centre de Recherche en Sciences Naturelles/Lwiro (LWI). Les doubles sont au Jardin Botanique National de Belgique (BR) et dans des diverses institutions universitaires et des recherches européennes.

La grande partie de la collection de ces expéditions fait partie de la flore d'Afrique Centrale (du PNKB en particulier) collectionnée dans différents Herbariums d'Afrique Centrale et de l'Europe. Bien que cette collection était déterminée jusqu'au rang spécifique par certains experts, bon nombre d'entre elle mérite encore une vérification. Le travail de Fischer (1996) effectué au PNKB montre que la richesse de la flore du PNKB est estimée à 1043 espèces provenant de 156 familles de plantes dont les Rubiaceae viennent en troisième position avec 46 espèces après les Orchidaceae (103 espèces) et les Asteraceae avec 74 espèces.

Les Rubiaceae sont des plantes très riche en espèces (Davis et Figueiredo, 2007 ; Lachenaud et al., 2013 ; Lachenaud, 2013), avec un nombre d'espèces estimé au niveau mondial à 13143 et environ 611 genres
(Davis et al., 2009). Ce sont des arbres, arbustes, lianes ou plantes herbacées, à feuilles opposées ou verticillées, généralement entières, à nervation pennée, à stipules interpétiolaires (Davis et Figueiredo, 2007). Leurs fleurs sont tétra ou penta mères, généralement hermaphrodites et actinomorphes (Lachenaud, 2013).

Les récoltes des Rubiaceae comme d'autres plantes dans le PNKB ont été enregistrées entre 1920 et 2013. Après l'indépendance de la RDC, il y a eu peu d'expédition surtout à l'Est du pays. Mais vers 2002, les récoltes ont recommencé timidement dans le PNKB suite aux travaux des chercheurs locaux à travers différents projets. Pour ce fait, la présente étude se fixe comme objectif, à contribuer à la connaissance de la flore des Rubiaceae et à la mise mettre à jour les données taxonomiques et écologiques (diversité et signalisation d'endémisme) de cette famille au PNKB seulement dans les trois sites ciblés. Pour ce travail, nous porterons l'attention sur la diversité (la richesse spécifique) et l'endémisme des Rubiaceae aux alentours et dans le PNKB et la comparaison entre les sites. Cet article s'intéresse aussi à la biodiversité de cette famille des Angiospermes.

Les études sur la répartition des espèces au niveau mondial montrent une distribution non uniforme sur toute l'étendue du globe (Sechrest et al., 2002), la forte diversité état concentrée sur des petites zones (Lachenaud, 2013). Les programmes de conservation de la biodiversité ont été orientés dans ces zones de haute diversité appelées le plus souvent «hotspots» (Myers et al., 2000 ; Lachenaud et al., 2013). Celles-ci sont déterminées en fonction de la richesse spécifique, du taux d'endémisme et du degré de vulnérabilité de l'habitat (Godefroid et al., 2011).

\section{MATÉRIEL ET MÉTHODES Milieu d'étude}

Situé à l'Est de la RDC, en province du Sud-Kivu dans le Rift albertin, le PNKB s'étend du bassin du fleuve Congo près d'Itebero-Utu jusqu'à sa frontière occidentale 
au Nord-Ouest de Bukavu (Figure 1). Ce Parc (Figure 1) tire son origine de 2 monts les plus élevés de ce massif forestier, Kahuzi (3326 m d'altitude) et Biega $(2790 \mathrm{~m})$. L'importance internationale du PNKB fut reconnue par l'UNESCO en 1980, comme le patrimoine mondial mais malheureusement en 1997 ; ce site a été placé sur la liste du patrimoine Mondial de l'UNESCO en péril (Mangambu et al., 2010).

Notre étude s'est beaucoup focalisée sur toute la partie montagnarde et une petite partie de la basse altitude du Parc (Itebero et Lulingu) sur base des informations disponibles dans les collections. Dans les parties basses du Parc, le climat peut être considéré comme uniformément chaud, aussi bien qu'au courant d'une journée qu'annuellement (Mangambu et al., 2013). Les précipitations sont très élevées, mais pas distribuées uniformément tout au long de l'année. On assiste plutôt à deux saisons des pluies séparées par des saisons sèches qui sont entre le mai et juin, l'autre entre les mois d'octobre et de décembre. La température moyenne annuelle s'élève entre 0,6 au sommet de mont Kahuzi, $18{ }^{\circ} \mathrm{C}$ dans la haute altitude et $20,5^{\circ} \mathrm{C}$ dans la partie de la plaine (Yamagiwa et al., 2005).

La végétation du PNKB est composée de six grandes formations végétales (Fischer, 1996). Elles se succèdent en fonction de l'altitude de la manière suivante: forêt ombrophile (750-1250 m) ; forêt de transition $(1250-1700 \mathrm{~m})$; forêt ombrophile de montagne (1700-2400 m) ; forêt de bambous (2400-2600 m) ; les marais et tourbières et enfin les bruyères subalpines (au-delà de $2600 \mathrm{~m})$.

\section{Analyse des matériels examinés au laboratoire}

Une banque des données provenant d'une collection de 1703 spécimens de plantes récoltés au PNKB et conservés dans les herbaria de Lwiro (LWI) et de Mulungu (MLGU) a été constituée. L'encodage s'est fait par le logiciel Brahms à partir des éléments de fiches d'herbier (date de récolte, numéro de récolte, nom de récolteur, nom scientifique, lieu de récolte ou (localité), altitude et l'habitat). L'ouvrage de Bamps (1982) a été utilisé pour certains échantillons où les coordonnées géographiques n'étaient pas signalées sur leurs étiquettes. Après, nous avons procédé à l'établissement d'une liste floristique des espèces récoltées au PNKB et conservées dans LWI et MLGU.

\section{Méthodologie utilisée pour le traitement}

Les méthodes classiques de taxonomie d'herbier et d'identification des collections d'herbier ont été suivies dans le cadre de cette étude (De Vogel, 1987 ; Lachenaud et al., 2013). Les fleurs et les fruits de certaines espèces ont été réhydratés dans l'alcool dilué jusqu'à $2 \%$ puis disséqués et observés à la loupe binoculaire afin de confirmer ou de corriger l'ancienne identification (Godefroid et al., 2011) et classé selon APG III, 2009. Enfin, l'ouvrage de Lebrun et Stork (1997) a été aussi utilisé pour la vérification et l'actualisation des noms scientifiques des espèces.

\section{Analyses des données sur la biodiversité}

Une multitude d'indices est développée dans les études de la biodiversité. Pour cette étude, les principales indices utilisés sont les suivants : indices de Shannon, de Piélou et de Jaccard (Margurran, 2004 ; Hammer et al., 2001 ; Grall et Hily, 2003).

- L'indice de diversité de Shannon (H'), 1949, repris par Grall et Hily, 2003 (indique la richesse en espèces au sein d'un écosystème local).

$H^{\prime}=-\sum\left[(N i / N) * \log _{2}(N i / N)\right]$,

avec $N i$ le nombre total d'individus d'une espèce donnée (où $i$ variant de 1 à $\Sigma$ ), $\Sigma$ le nombre total d'espèces et $N$ le nombre total d'individus. Lorsque $H^{\prime}=0$, tous les individus du peuplement appartiennent à une même espèce. H' est minimal lorsque dans un peuplement chaque espèce est représentée par un seul individu. H' est maximal quand tous les individus sont repartis de façon égale sur toutes les espèces. 


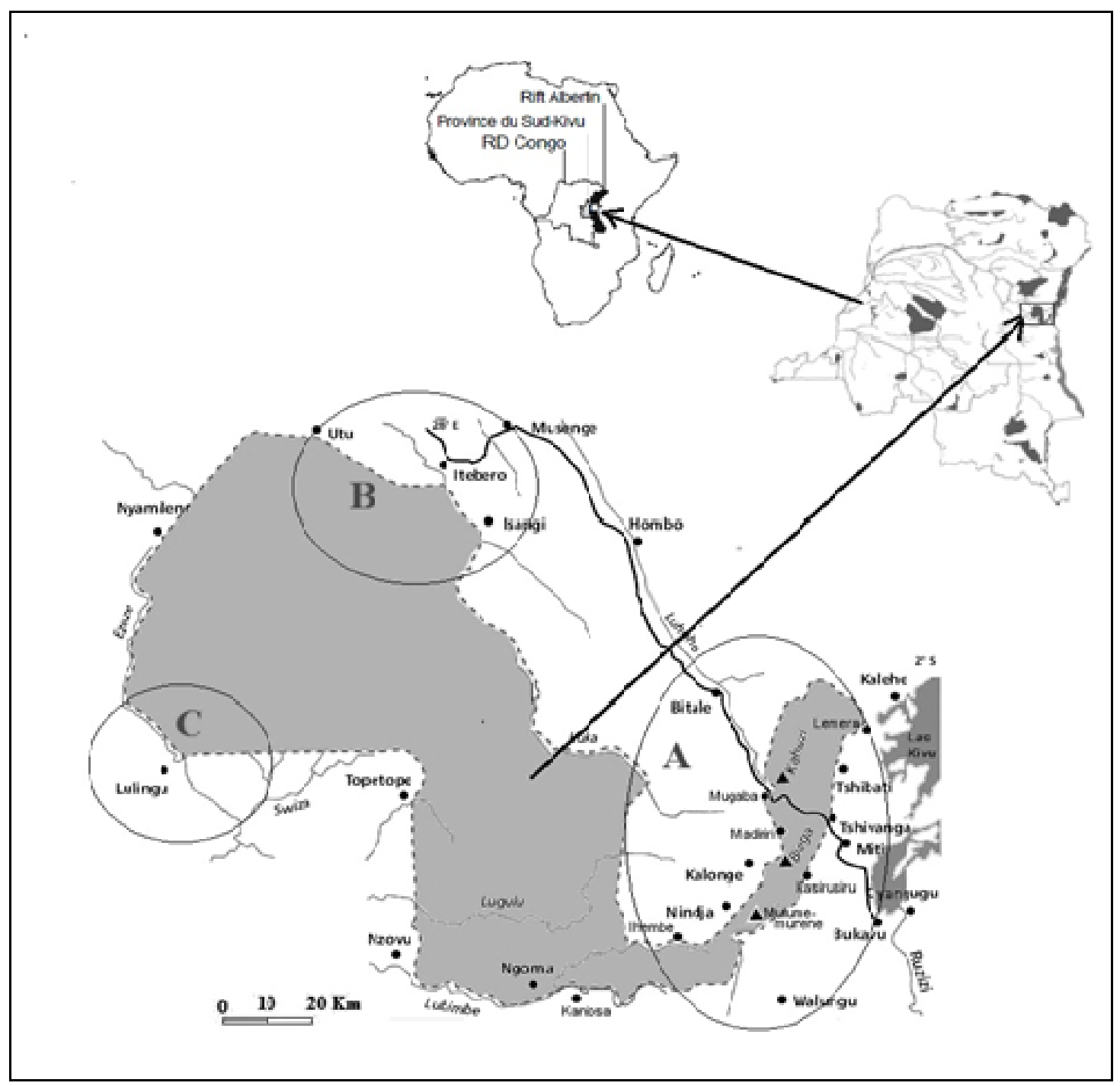

Figure 1: Localisation des sites d'échantillonnages dans le PNKB. Les zones encerclées représentent les sites échantillonnés. A : Partie montagnarde, B : Itebero dans la partie basse altitude et C : Lulingu «basse altitude » qui couvrent $\pm 2 / 6$, soit $2400 \mathrm{~km} »)$.

- L'équitabilité de Piélou (EQ) est le rapport de la diversité d'un peuplement ou d'un échantillon et le nombre $\mathrm{N}$ d'espèces présentes. Il exprime la régularité, la répartition équitable des individus au sein des placettes entre les espèces (Grall et Hily, 2003).

$$
E Q=I S H / \log _{2}(N)
$$

- Le coefficient de Jaccard $(I J)$ est un indice de similarité dans la composition des espèces entre les différents habitats qui consiste à traiter des données binaires de présenceabsence (1-0), notamment d'espèces sur des parcelles d'une communauté végétale.

Dans cet indice, (a) représente le nombre d'espèces présentes dans les deux parcelles, tandis que $b$ et $c$, le nombre d'espèces uniquement présentes respectivement dans la première parcelle et la deuxième parcelle. Cet indice a été calculé pour estimer la ressemblance entre les sites étudiés sur base des abondances et de richesse spécifiques. 
Les principales statistiques employées pour comparer les données ont été faites par les logiciels Past et $\mathrm{R}$ respectivement pour calculer la diversité, la similarité (Hammer et al., 2001) et le test $t$ de Student. Ce test sert à comparer les moyennes de deux séries de données indépendantes. Pour calculer la formule classique du $t$ de Student (c'est-à-dire pour des données indépendantes), il faut calculer les moyennes de chaque série et la variance commune des deux séries réunies (Ponger, 2008) ; ensuite on calcule $t$ :

$$
t=\frac{\overline{\mathbf{X}}_{1}-\overline{\mathbf{X}}_{2}}{\sqrt{\frac{S_{p}^{2}}{n_{1}^{2}}+\frac{S_{p}^{2}}{n_{2}}}}
$$

avec $\bar{X}_{1}$ et $\bar{X}_{2}:$ les moyennes arithmétiques des deux séries. $S_{p}^{2}$ : Variance commune des deux séries et $n_{1}$ et $n_{2}$ sont les nombres de données dans les deux séries (Ponger, 2008).

A comme l'hypothèse nulle $\mathrm{H}_{\mathrm{O}}$, ce test vérifie si les deux moyennes sont égales. Dans le cas contraire, l'hypothèse $H_{1}$ vérifie si les deux moyennes sont différentes au seuil de probabilité $(\mathrm{p}<0,005)$ par rapport à leur degré de liberté (n-1). C'est-à-dire en comparant le $t$ obtenu à la table des valeurs critiques de la distribution de $t$ (Raufaste, 2013).

\section{RÉSULTATS}

Diversité et connaissance taxonomique

Un total de 63 espèces, 13 infraspécifiques $(10$ sous-espèces et 3 variétés) regroupées dans 41 genres et 18 inconnues a été inventorié (Tableau 1). Cinq genres de cette famille ont été les plus représentés en espèces. Le genre Psychotria est le mieux représenté avec $11,7 \%$ suivi de Rytigynia, Tricalysia, Pavetta et Tarenna; respectivement avec $7,4 \% ; 7,4 \% ; 6,4 \%$ et $5,3 \%$. Dix genres (Amaralia, Diodia, Empogona, Galiniera, Hymenodictyon, Keetia, Leptactinia, Nauclea, Sericanthe et Tarenna) et douze infraspécifiques
(Aulacocalyx jasminiflora Hook. F. subsp. kivuensis E. Figueiredo; Lasianthus kilimadscharicus K. Schum. subsp. hirsutus Jannerup ; Otiophora pauciflora Bak. subsp. pauciflora; Pseudosabicea arborea (Schum.) Halle subsp. bequaertii (De Wild.) Verdc.; Psychotria chalconeura (K. Schum.) E.M.A. Petit var. montana E.M.A. Petit ; Psychotria mahonii C.H. Wright var. puberula (E.Petit) Verdc.; Rutidea smithii Hiern subsp. submontana (K. Krause) Bridson ; Rytigynia bagshawei (S. Moore) Robyns var lebrunii (Robyns) Verdc.; Rytigynia bridsoniae Verdc. subsp. kahurica Verdc.; Sericanthe leonardii (N. Hallé) Robbr. subsp. leonardii ; Sericanthe leonardii (N. Hallé) Robbr. subsp. venosa Robbr. et Tarenna pollidula subsp. pollidula) ont été ajoutés sur la liste floristique de Fischer (1996).

\section{Habitat et écologie des Rubiaceae du PNKB}

Suivant leur écologie, les Rubiaceae du PNKB sont de plantes de sous-bois et occupent une assez grande variété d'habitats, à l'exclusion de celles qu'on retrouve dans les sommets des Mont Kahuzi et Biega. Trois catégories principales peuvent être distinguées: les espèces de sous-bois, les espèces forestières héliophiles et les espèces de marais (Figueiredo, 2008). Les principaux facteurs affectant la distribution des Rubiaceae dans les sous-bois au PNKB semblent être la lumière et l'humidité. L'importance de ce dernier facteur se manifeste par la prédilection qu'ont de nombreuses espèces pour les bas-fonds et abords de ruisseaux. Le fait est plus marqué dans les forêts de plaine que dans les régions montagnardes, où l'humidité permanente permet à certaines espèces habituellement hydrophiles de pousser sur la terre ferme.

La majorité des espèces héliophiles sont des espèces lianescentes et la plupart des espèces arborescentes. Ces espèces sont limitées aux lisières forestières à fort ensoleillement. Certaines sont strictement 
liées aux formations ripicoles, où elles croissent avec les pieds dans l'eau. Les autres, sont des plantes pionnières colonisant les clairières et les bords de pistes, où elles peuvent être très abondantes ; on peut aussi les observer sur les lisières où elles forment un front pionnier. Suite à l'invention de Sericostachys scandens au PNKB, les Rubiaceae sont moins récoltées car la strate arborescente devient faible ou presqu'absente. C'est par exemple le cas de R16 dans la station de Tshivanga.

De nombreuses espèces sont caractérisées par la présence de bactéries symbiotiques dans leurs feuilles. Ces symbioses foliaires se rencontrent également chez d'autres groupes de plantes, mais elles ont été relativement peu étudiées en comparaison avec les symbioses racinaires. Il en existe deux types : la symbiose foliaire nodulante et la symbiose foliaire non nodulante. . C'est pour cette raison que, ces espèces des Rubiaceae s'adaptent dans plusieurs biotopes.

\section{Détermination des indices de biodiversité et similarité entre sites prospectés}

Les résultats obtenus montrent que les indices de diversité effectués confirment la diversité des Rubiaceae dans le PNKB. L'indice de Shannon calculé est plus élevé dans les sites R6, R2, R8 et R15 que dans les sites R5 et R10. Cependant, l'équitabilité de Piélou calculée varie entre 0,9 à 1 (Tableau 2). Ces fortes valeurs (proches de 1 ou équivalent à l'Unité) reflètent une distribution équitable des individus entre les espèces de Rubiaceae sur le massif du PNKB.

Les sites forment deux blocs suivant qu'ils sont de la basse ou de la haute altitude. Pour les sites de la haute altitude, il s'observe un niveau de regroupement suivant les sites de récolte (Figure 2). La comparaison de la moyenne par le test de Student (Figure 4) montre une différence significative $(\mathrm{t}=$ 7.6442 , df $=112.587$, p-value $=7.599 \mathrm{e}-12$ ) entre les espèces de la haute et de la basse altitude sur base des abondances et de la richesse spécifique.

Selon les informations recueillies sur les fiches de récoltes des chercheurs, les résultats montrent issus des analyses de similarité affectées que les Rubiaceae sont beaucoup plus diversifiées dans les sites R6, $\mathrm{R} 2, \mathrm{R} 8$ et R15 tandis que sur les sites R5 et R10, elles sont moins diversifiés (Figure 2). Mais, la richesse spécifique élevée dans notre milieu d'étude s'explique en partie par la diversité des habitats. C'est ce qui s'est observé dans les milieux (R11 et R14) représentant dans ce travail les marais Lushadja et Musisi et montrant qu'il n'y a pas eu plusieurs spécimens en provenance de ces endroits (Figure 3). Ceci montre que les Rubiaceae ne sont pas des plantes des marais, mais elles abondent les milieux hydromorphes R7 et R12).

\section{Endémisme des Rubiaceae dans le PNKB}

Le PNKB regorge environ 10 espèces de Rubiaceae endémiques dans le RiftAlbertin (Ntore, 2008 ; Robbrecht, 1996a) parmi lesquelles 3 sont uniquement endémiques du PNKB : Pauridiantha kahuziensis Ntore, Psychotria bugoyensis K. Krause et Sericanthe leonardii (N. Hallé) Robbr. subsp. leonardii. L'espèce Psychotria bugoyensis $\mathrm{K}$. Krause a été plus abondante et avec une large distribution dans le PNKB; elle a été récoltée dans 4 milieux différents (Mugaba, Kahuzi, Kasirusiru et au Mont Biega) tandis que Pauridiantha kahuziensis Ntore se retrouve uniquement au Mont Kahuzi et Sericanthe leonardii (N. Hallé) Robbr. subsp. leonardii à Musenyi (Mont Kahuzi). 
Tableau 1 : Check-list des espèces (63 espèces, 13 infraspécifiques et 18 inconnues et 12 espèces ont été ajoutés sur la liste floristique de Fischer, 1996).

\begin{tabular}{|c|c|c|c|}
\hline Check-list des espèces & Z.P & Z.M. & Pr \\
\hline Aidia micrantha (K.Schum.) Bull. & + & - & 1 \\
\hline Amaralia scheibouriae (Kost. f.) Wern. & + & + & 2 \\
\hline Anthospermum usambarense K. Schum. & - & + & 1 \\
\hline Aulacocalyx jasminiflora Hook. F. subsp. kivuensis E. Figueiredo & + & - & 1 \\
\hline Bertiera dewevrei De wild. \& T. Durand & + & + & 2 \\
\hline B. racemosa (G. Don) K. Schum. & + & - & 1 \\
\hline Canthium sp I & + & + & 2 \\
\hline Canthium sp II & + & + & 2 \\
\hline Chassalia cristata (Hiern) Bremek. & - & + & 1 \\
\hline C. parviflora K. Schum. & - & + & 1 \\
\hline C. subochreata (De Wild.) Robyns & + & + & 2 \\
\hline Coffea eugenioides Moore & + & + & 2 \\
\hline Craterispermum laurinum Benth. & + & + & 2 \\
\hline Diodia sarmentosa $\mathrm{Sw}$. & + & + & 2 \\
\hline Empogona aulacosperma (Robbr.) J. Tosh \& Robbr. & + & + & 2 \\
\hline Galiniera saxifraga (Hochst.) Bridson & + & + & 2 \\
\hline Galium aparinoides Forssk. & + & + & 2 \\
\hline G. spurium L. & - & + & 1 \\
\hline Geophila obvollata (Schumach.) F. Didr. & + & - & 1 \\
\hline G. repens (L.) I.M. Johnston & + & - & 1 \\
\hline Hallea rubrostipulata K. Schum. (K. Schum.) Leroy & + & + & 2 \\
\hline Hedythyrsus thamnoideus (K. Schum.) Bremek. & - & + & 1 \\
\hline Hymenodictyon floribudum (Hochst. ex Steud) B.L. & + & + & 2 \\
\hline Ixora burundiensis Bridson & - & + & 1 \\
\hline Ixora. sp. & - & + & 1 \\
\hline Keetia angustifolia Bridson & - & + & 1 \\
\hline Keetia gueinzii (Sond.) Bridson & - & + & 1 \\
\hline Lasianthus kilimadscharicus K. Schum. & - & + & 1 \\
\hline L. kilimandscharicus K. Schum. subsp. hirsutus Jannerup & - & + & 1 \\
\hline Leptactinia platyphylla (Hiern) Wernh. & - & + & 1 \\
\hline Mussaenda polita Hiern & + & - & 1 \\
\hline Nauclea vanderguchtii (De Wild.) Petit & + & - & 1 \\
\hline Oldenlandia herbacea (L.) Roxb. & + & + & 2 \\
\hline Otiophora pauciflora Bak. subsp. burtii (Milne-Redh.) Verdc. & - & + & 1 \\
\hline O. pauciflora Bak. Subsp. Pauciflora & - & + & 1 \\
\hline Oxyanthus speciosus DC. & - & + & 1 \\
\hline O. troupinii Bridson & - & + & 1 \\
\hline Pauridiantha dewevrei (De Wild.) Bremek. & - & + & 1 \\
\hline P. kahuziensis Ntore* & - & + & 1 \\
\hline P. paucinervis (Hiern) Bremek. & - & + & 1 \\
\hline P. pierlotii N. Hallé & - & + & 1 \\
\hline Pausynistalia sp. & + & - & 1 \\
\hline Pavetta ankolensis Bridson & - & + & 1 \\
\hline P. pierlotii Bridson & - & + & 1 \\
\hline P. rupifilis Drem. & - & + & 1 \\
\hline P. rwandensis Bridson & - & + & 1 \\
\hline Pavetta sp. I & - & + & 1 \\
\hline
\end{tabular}




\begin{tabular}{|c|c|c|c|}
\hline Pavetta sp. II & - & + & 1 \\
\hline Pentas longiflora Oliv. & - & + & 1 \\
\hline P. zanzibarica (Klotzsch) Vatke & - & + & 1 \\
\hline Pseudosabicea arborea (Schum.) Halle subsp. bequaertii (De Wild.) Verdc. & - & + & 1 \\
\hline Psychotria bugoyensis K. Krause* & - & + & 1 \\
\hline P. chalconeura (K. Schum.) E.M.A. Petit var. montana E.M.A. Petit & - & + & 1 \\
\hline P. leonardiana E.M.A. Petit & + & + & 2 \\
\hline P. mahonii C.H. Wright C. H. Wright & + & + & 2 \\
\hline P. mahonii C.H. Wright var. puberula (E.Petit) Verdc. & - & + & 1 \\
\hline P. palustris Petit & - & + & 1 \\
\hline P. parvistipulata E.M.A. Petit & - & + & 1 \\
\hline Psychotria sp. I & - & + & 1 \\
\hline Psychotria sp. II & - & + & 1 \\
\hline Psychotria sp. III & - & + & 1 \\
\hline Psychotria sp. IV & - & + & 1 \\
\hline Rothmannia whitfieldii (Lindl.) Dandy & + & - & 1 \\
\hline Rubia cordifolia $\mathrm{L}$. & - & + & 1 \\
\hline Rutidea odorata Krause & - & + & 1 \\
\hline$R$. orientalis Bridson & - & + & 1 \\
\hline R. smithii Hiern & - & + & 1 \\
\hline R. smithii Hiern subsp. submontana (K. Krause) Bridson & - & + & 1 \\
\hline Rytigynia bagshawei (S. Moore) Robyns & - & + & 1 \\
\hline R. bagshawei (S. Moore) Robyns var lebrunii (Robyns) Verdc. & - & + & 1 \\
\hline R.beniensis (De. Wild.) Robyns & - & + & 1 \\
\hline R. bridsoniae Verdc. subsp. kahurica Verdc. & - & + & 1 \\
\hline R. bugoyensis (K. Krause) Verdc. & - & + & 1 \\
\hline R. castanea Robyns & - & + & 1 \\
\hline Rytigynia sp. & - & + & 1 \\
\hline Sericanthe leonardii (N. Hallé) Robbr. subsp. leonardii* & - & + & 1 \\
\hline S. leonardii (N. Hallé) Robbr. subsp. venosa Robbr. & + & + & 2 \\
\hline Spermacoce princeae (Schum.) Verdc. & - & + & 1 \\
\hline Tarenna gilletii (De Wild. \& Th. Dur.) N. Hallé & - & + & 1 \\
\hline Tarenna inops Degreef & - & + & 1 \\
\hline T. kivuensis Degreef & - & + & 1 \\
\hline T. pollidula subsp. pollidula & - & + & 1 \\
\hline Tarenna sp. & + & + & 2 \\
\hline Tricalysia kiwuensis (K. Krause) Robyns & - & + & 1 \\
\hline T. niamniamensis C.H.Weint \& Hiern & - & + & 1 \\
\hline T. ruandensis Bremek. & - & + & 1 \\
\hline Tricalysia sp. & - & + & 1 \\
\hline Tricalysia sp. I & - & + & 1 \\
\hline Tricalysia sp. II & - & + & 1 \\
\hline Tricalysia vanroechoudtii (Lebrun ex Van Roech.) Robbr. & - & + & 1 \\
\hline Virectaria major (K. Shum.)Verdc. & + & + & 1 \\
\hline Rubiaceae inconnue I & - & + & 1 \\
\hline Rubiaceae inconnue II & - & + & 1 \\
\hline Rubiaceae inconnue III & - & + & 1 \\
\hline Total & 27 & 85 & \\
\hline
\end{tabular}


Tableau 2 : Analyse de diversité des sites (L'analyse de la ressemblance entre les sites a montré un regroupement des espèces suivant l'altitude sur base de présence/absence des espèces).

\begin{tabular}{lcccccccccccccccc}
\hline & R1 & R2 & R3 & R4 & R5 & R6 & R7 & R8 & R9 & R10 & R11 & R12 & R13 & R14 & R15 & R16 \\
\hline Taxa_S & 43 & 46 & 36 & 35 & 18 & 47 & 22 & 40 & 27 & 19 & 23 & 31 & 31 & 29 & 42 & 38 \\
Individuals & 112 & 102 & 108 & 94 & 111 & 146 & 94 & 110 & 94 & 101 & 97 & 94 & 108 & 106 & 126 & 100 \\
Shannon_H & 3,6 & 3,6 & 3,3 & 3,4 & 2,7 & 3,7 & 2,8 & 3,6 & 3,1 & 2,5 & 2,9 & 3,3 & 3,1 & 3,2 & 3,6 & 3,5 \\
Equitability_J & 1 & 0,9 & 0,9 & 0,9 & 0,9 & 1 & 0,9 & 1 & 0,9 & 0,9 & 0,9 & 0,9 & 0,9 & 0,9 & 1 & 1 \\
\hline
\end{tabular}

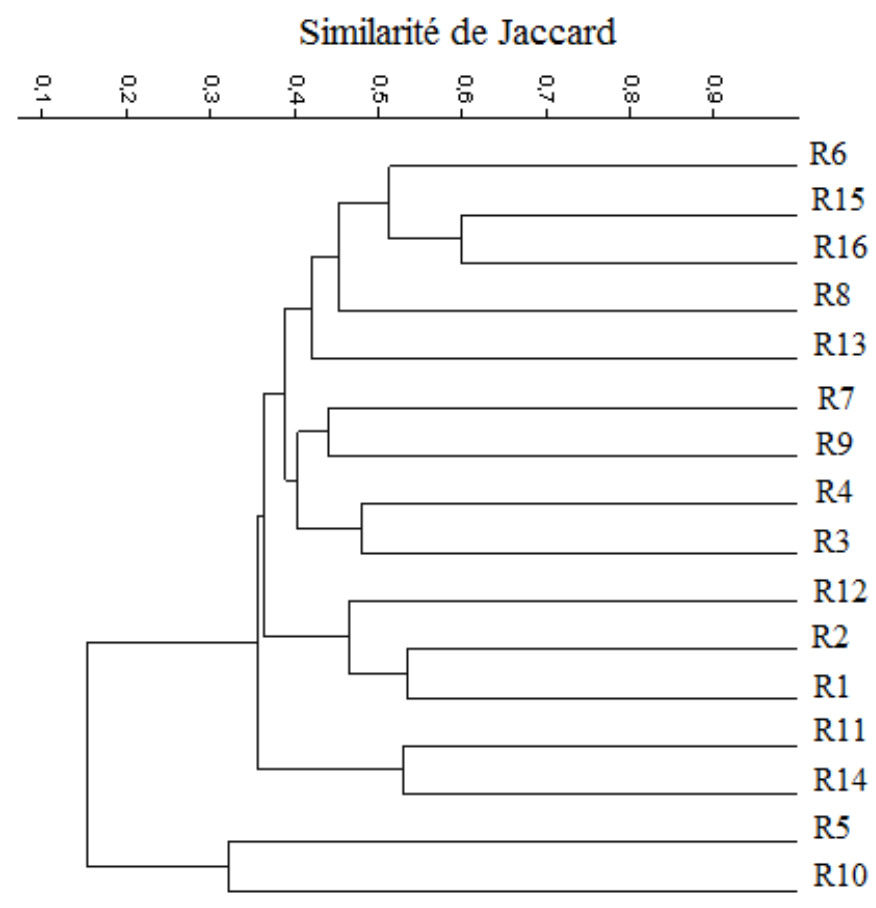

Figure 2 : Similarité des sites par la méthode de Jaccard. (La comparaison de la moyenne par le test de Student $(t)$ entre les espèces de la haute et de la basse altitude sur la base des abondances et de la richesse spécifique). 


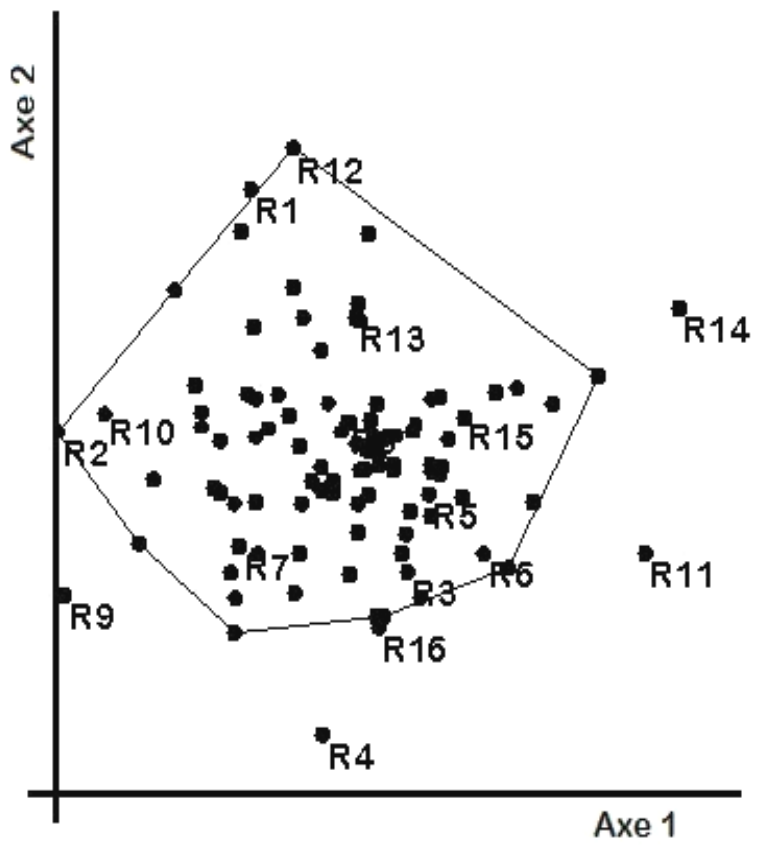

Figure 3 : Correspondance des sites prospectés (regroupement suivant les sites des récoltes). R1: Bugulumisa; R2: Musenyi ; R3 : Mugaba; R4: Ironga ; R5: Itebero ; R6: Kahuzi; R7: Karashomwa; R8 : Kasirusiru ; R9 : Lemera ; R10 : Lulingu ; R11 : Lushadja ; R12 : Madiriri ; R13 : Biega ; R14 : Musisi ; R15 : Tshibati et R16 : Tshivanga

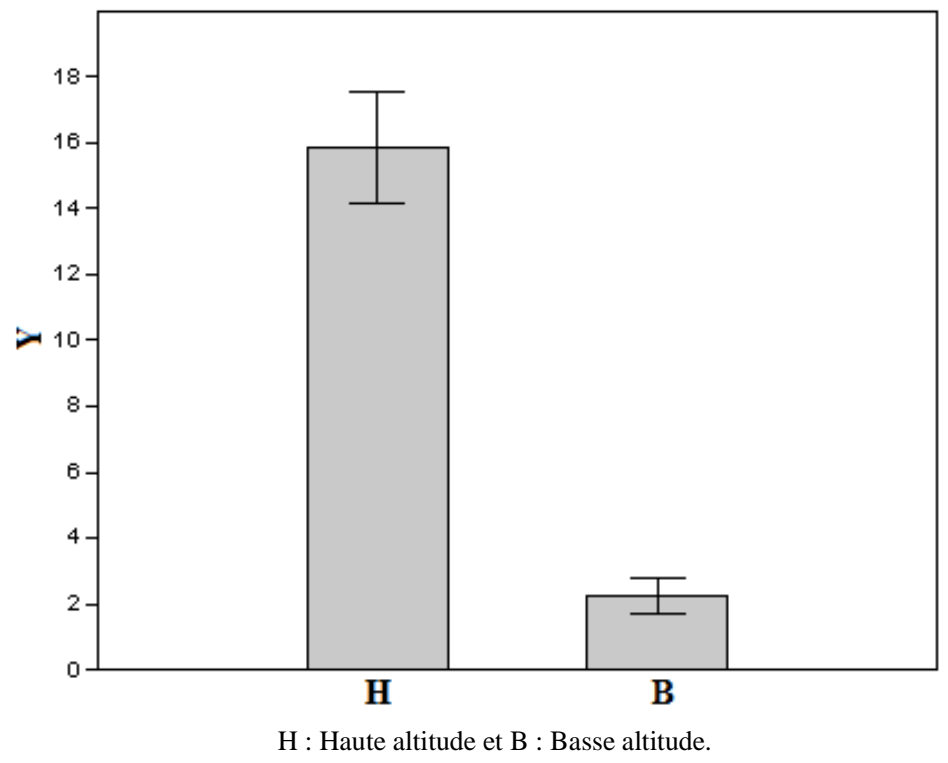

Figure 4 : Différence des abondances entre la haute et la basse altitude (La richesse spécifique élevée dans la partie haute altitude s et moindre dans la partie basse altitude, station de Lulungu et Itebero). 


\section{DISCUSSION}

\section{Diversité et endémisme des Rubiaceae}

Les Rubiaceae sont parmi les plantes qui ont une très grande diversité dans le monde tropicale (Davis et al., 2009; Lachenaud et Jongkind, 2010; Lachenaud et al., 2013). Ces plantes sont très répandues et se trouvent dans toutes les grandes régions du monde, sauf dans l'Antarctique. Elles sont surtout un groupe des plantes sous les tropiques avec la plus grande diversité dans les zones de basse et moyenne altitudes des forêts humides (Ntore, 2008 ; Davis et al., 2009). Pour ces auteurs, au sein de cette famille, le genre Psychotria a un plus grand nombre d'espèces (1834) et occupe la première place dans la famille sur le plan mondial (Robbrecht, 1996a; Lachenaud, 2013). Les résultats de notre étude montrent aussi la richesse spécifique du genre Psychotria (11 espèce), l'amenant à occuper ainsi la première position dans cette étude. Figueiredo (2008) a aussi observé la prédominance du genre Psychotria (35 espèces) lors d'une étude sur les Rubiaceae d'Angola.

En comparant notre flore avec la flore du Rwanda sur le plan richesse taxonomique, nous constatons que la flore du PNKB est riche avec 63 espèces et 41 genres par rapport à l'inventaire effectué sur toute la flore du Rwanda (120 espèces et seulement 42 genres) par Troupin, 1985. Dans plusieurs travaux récents sur la distribution et la diversité des groupes taxonomiques en Afrique, l'hypothèse sur l'existence des refuges forestiers est couramment évoquée (Robbrecht, 1996a ; Stevart, 2003 ; Lachenaud, 2013). Cette hypothèse stipule que pendant les dernières périodes glaciaires du Pléistocène, l'Afrique a connu une baisse de température et de pluviométrie. La végétation des forêts a été fortement perturbée et réduite au profit des savanes; les végétaux ont survécu dans les zones où les conditions de pluviométrie et de température permettaient leur maintien (Maley, 1996 ; Lachenaud, 2013). Par la suite, les espèces se seraient dispersées à partir de ces zones appelées refuges forestiers en colonisant des habitants nouveaux. Par conséquent, la richesse spécifique et le taux d'endémisme devraient être élevés dans ces sites (Lachenaud et al., 2013). On devrait s'attendre à avoir un gradient de diversité à partir des zones refuges. Pour bien vérifier cette hypothèse de nos prédécesseurs, une multitude de descente sur le terrain serait souhaitable enfin de confirmer ou d'infirmer.

Certains auteurs comme Halle (1966) et Robbrecht (1996b) démontrent que les Rubiaceae seraient diversifiées dans les forêts à fortes pluviosité et auraient migré vers les zones plus arides avec acquisition de caractères nouveaux et adaptations à de nouvelles conditions écologiques. Nous appuyons cette idée à partir de l'espèce Pauridiantha kahuziensis Ntore (Ntore, 2008) qui se retrouve pour le moment à un seul point au Mont Kahuzi dans le PNKB, car l'espèce est vraisemblablement un vestige d'une population jadis abondante et largement distribuée. Depuis 1959 où l'espèce a été récoltée par Léonard (Léonard, A. 4802 (BR \& YBI) jusqu'aujourd'hui, seulement quelques pieds ont pu être observés sur le même mont, formant ainsi une petite colonie.

\section{Occurrence de récolte des Rubiaceae au PNKB}

Les résultats floristiques entre la végétation de la plaine et celle de la zone de montagne présentent une richesse spécifique élevée dans la partie haute altitude (85 espèces, soit 90,4\%) par rapport à celle de la zone de plaine qui est représentée par 27 espèces (soit, 28,7\%) de la diversité totale de la flore des Rubiaceae inventoriées (Figure 4 et Tableau 1). Cette pauvreté de la diversité dans cette zone de basse altitude peut être expliquée par deux raisons : cette zone n'a pas été bien exploitée par rapport à celle des montagnes qui a été bien fouillé pendant des grandes explorations botanique en RDC. Et son habitat est en général riche en espèce que celui de la plaine (Plumptre et al., 2007). 
Un grand nombre d'espèces enregistrées dans cette étude a été récolté aux endroits secs et humides, mais moins abondantes dans les marais et au bord des cours d'eau. Cela a été aussi confirmé par Troupin (1985) dans la flore du Rwanda. La même chose a été prouvée par Lachenaud (2013) qui avait aussi remarqué que les espèces liées aux habitats de terre ferme sont plus nombreuses et plus récoltées que celles qui se développent sur les sols hydromorphes et dans les milieux aquatiques. Ces résultats prouvent que les herbaria de la région renferment une information très riche sur la biodiversité de la région du Rift-Albertin en général et celle du PNKB en particulier surtout sur la famille des Rubiaceae dont on fait allusion dans ce travail. Une étude très étendue dans la partie basse altitude du PNKB où nous n'avons pas eu assez d'informations est indispensable afin de compléter cette étude que nous considérons encore comme préliminaire.

Pour mieux comprendre cette occurrence de récolte des Rubiaceae, nous comparons nos données avec les données des autres sites proches du PNKB qui se trouvent dans le système montagneux KivuRuwenzori. Dans le Parc de Nyungwe, les travaux de Dowsett-Lemaire (1990) montrent une diversité de 59 espèces et 19 genres différents. Dans la crête du Rwanda, Habiyaremye (1997) a récolté 53 espèces des Rubiaceae appartenant à 25 genres. En RDC dans les régions de Misotshi-Kabogo sur le Mont Marungu à l'Est de la RDC, Plumptre et al. (2008) ont récolté 102 espèces dont 53 genres.

Ces résultats montrent une affinité floristique prononcée entres les sites qui se trouvent dans le Rift-Albertin. Cela peut être expliqué par le fait que ces sites sont tous dans les systèmes montagneux KivuRuwenzori qui est réputé riche en phytodiversité (Plumptre et al, 2007 ; Plumptre et al., 2008; Mangambu et al., 2013).

Autre raison est d'ordre paléoenvironnemental, la large diversité en espèces au sein du système Kivu-Ruwenzori peut être expliquée par sa couverture végétale qui change continuellement, en réponse à des variations macroclimatiques et microclimatiques (Sonké et al., 2012). Il s'agit-là de la suite de la stabilité climatique des temps géologiques passés (Myers et al., 2000 ; Maley, 2001). Cela grâce aux conditions climatiques favorables aux plantes vasculaires, pouvant conserver des spores et des grains de pollen capables de germer après plusieurs années (Runge, 2007).

Plusieurs études ont souligné l'importance des fluctuations climatiques du Pléistocène et de l'Holocène pour expliquer la distribution actuelle des plantes dans ce système montagneux congolais, un territoire marqué, en ce qui concerne la chronologie historique des événements climatiques, par l'alternance des périodes froides et chaudes (Runge, 2007). Ces variations climatiques cycliques ont eu une grande influence sur la diversité des plantes et sur l'évolution du couvert végétal à l'Est de la RDC et d'autres pays d'Afrique centrale (Runge, 2007).

Les résultats obtenus confirment l'hypothèse de White (1993) qui annonce que les aires de distributions des Rubiaceae sont très prononcées dans les montagnes africaines de l'Est du continent (Myers et al., 2000 ; Sonké et al., 2012). Les mêmes résultats montrent que la connaissance de la flore de la RDC reste encore fragmentaire malgré plus d'un siècle de multiples explorations et de récoltes végétales réalisées. Ainsi, nous suggérons que les chercheurs locaux soient soutenus financièrement, pour enfin réaliser de multiples récoltes de plantes en général et de Rubiaceae en particulier surtout dans la partie de basse altitude vu le nombre réduit d'espèces dans cette partie du Parc inventorié dans les collections. Ceci dans le but de combler certaines lacunes sur la flore congolaise.

\section{REMERCIEMENTS}

Nous remercions les responsables du PNKB et tous les curateurs, employés, 
gestionnaires ou techniciens d'herbarium (LWI et MLGU). Qu'ils trouvent dans ces lignes nos remerciements les plus chaleureux.

\section{RÉFÉRENCES}

APG III. 2009. An update of the Angiosperm Phylogeny Group classification for the orders and families of flowering plants : APG III. J. Linn. Soc. Bot., 161: 105-121.

Bamps P. 1982. Répertoire des Lieux de Récolte. Jardin Botanique National de Belgique: Meise.

Davis AP, Figueiredo E. 2007. A checklist of the Rubiaceae (coffee family) of Bioko and Annobon (Equatorial Guinea, Gulf of Guinea). System. Biod. Jornal., 5(2): 159-186.

Davis AP, Govaerts R, Diane M, Bridson M, Ruhsam M, Moat J, Brummitt A. 2009. A global assessment of distribution, diversity, endemism and taxonomic effort in the Rubiaceae. An. Miss. Bot. Garden, 96(1): 1-11.

De Vogel F. 1987. Manuel of Herbarium Taxonomy: Theory and Practice. Ed. UNESCO, Regional Office for Sciences and Technology: Indonesia.

Dowsett-Lemaire. 1990. Physionomie et végétation de la forêt de Nyungwe, Rwanda. Rapport de Jupille, Liège, Belgique.

Figueiredo E. 2008. The Rubiaceae of Angola. J. Linn. Soc., Bot., 156: 537-638.

Fischer E. 1996. Die Vegetation des Parcs National de Kahuzi-Biega, Sud-Kivu, Zaïre. Franz Steiner Verlag : Stuttgart.

Habiyaremye M. 1997. Etude phytocoenologique de la dorsale orientale du lac Kivu (Rwanda). Anale des sciences économiques, 24, Musée Royal de l'Afrique centrale, Tervuren.

Hallé N. 1966. Famille des Rubiaceae ( 1 ère partie). Flore du Gabon, 12: 1-277.

Grall J, Hily C. 2003. Traitement des données stationnelles (faune). Fiche-technique.

Godefroid S, van de Vyver A, Stoffelen P, Robbrecht E, Vanderborght T. 2011. Testing the viability of seeds from old herbarium specimens for conservation purposes. Taxon, 60: 565-569.

Lebrun J-P, Stork A. 1997. Enumération des Plantes à Fleurs d'Afrique Tropicale Gamopétales : Clethraceae à Lamiaceae (vol. IV). Editions des Conservatoires et Jardins Botaniques de la ville de Genève : genève.

Hammer O, Harper D, Ryan P. 2001. PAST: Paleontological Statistics software package for education and data analysis. Paleontol. Elect., 4(1): 1-9.

Lachenaud O, Jongkind C, 2010. Three new or little-known Chassalia (Rubiaceae) species from West and Central Africa. Nord. J. Bot., 28: 13-20.

Lachenaud O, Droissart V, Dessein S, Stévart T, Simo M, Lemaire B, Taedoumg H, Sonké B. 2013. New records for the flora of Cameroon, including a new species of Psychotria (Rubiaceae) and range extensions for some rare species. $\mathrm{Pl}$. Ecol. Evol., 146(1/3): 121-133.

Lachenaud O. 2013. Le genre Psychotria (Rubiaceae) en Afrique occidentale et centrale: taxonomie, phylogénie et biogéographie/The genus Psychotria (Rubiaceae) in West and Central Africa: taxonomy, phylogeny and biogeography. Thèse de Doctorat, Fac. Sciences, U.L.B/Belgique, p 1121.

Maley J. 2001. La destruction catastrophique des forêts d'Afrique centrale survenue il y a environ 2500 ans exerce encore une influence majeure sur la répartition actuelle des formations végétales. Syst. Geo. Plant., 71(2): 777-796.

Margurran AE. 2004. Measuring Biological Diversity. Blackwell Publishing Company: United Kingdom.

Mangambu M, Habiyaremye M, Lina A, Ntahobavuka H. 2010. L'importance du groupement à Cyathea manniana dans la biodiversité du Parc National de Kahuzi Biega, R.D. Congo. Geo-Eco-Trop., 34(1/2): 45-63.

Mangambu M, Diggelen R, Mwanga Mwanga I, Ntahobavuka H, Robbrecht E. 2013. Espèces nouvellement signalées pour la 
flore Ptéridologique de la République Démocratique du Congo. Int. J. Biol. Chem. Sci., 7(1): 107-124.

Myers N, Mittermeier RA, Mittertneier CG, Da-Fonseca GAB, Kent J. 2000. Biodiversity hotspots for conservation priorities. Nature, 403: 853-858.

Ntore S. 2008. Révision $d u$ Genre Afrotropical pauridiantha (Rubiaceae) (Vol 15). National Botanic Garden of Belgium: Meise, Belgique.

Plumptre AJ, Eilu G, Ewango C, Ssegawa P, Nkuutu D, Gereau R, Beentje H, Poulsen AD, Fischer E, Goyder D, Pearce TR, Hafashimana D. 2007. The biodiversity of the Albertine Rift. Biol. Cons., 134: 178-194.

Plumptre AJ, Kujirakwinja D, Matanguru J, Kahindo C, Kaleme P, Marks B, Huhndorf M. 2008. Inventaires biologiques des régions de MisotshiKabogo (Mont Marungu à l'est de la RDC. Albertine Rilf Technical Reports. Unpublished report.

Ponger L. 2008. Variables Quantitatives : la Moyenne Estimations, Intervalle de Confiance et Tests Statistiques Régulation et Dynamique des Génomes; Musée National d'Histoire Naturelle : Paris.

Raufaste E. 2013. Interpréter des Résultats de $\mathrm{t}$ de Student à Echantillons Appariés. Analyses Statistique, Losange.

Robbrecht E. 1996a. Geography of African Rubiaceae with reference to glacial rain forest refuges. In The Biodiversity of African Plants, van der Maesen LJG, van der Burgt XM , van Madenbach de Rooy JM (eds). Kluwer: Dordrecht; 564-581.

Robbrecht E. 1996b. Genetic distribution patterns in sub-Saharan African Rubiaceae (Angiospermae). J. Biog., 23: 311-328.

Runge J. 2007. Des déserts et des forêts, histoire du paysage et du climat de l'Afrique Centrale au Quaternaire Supérieur. Geo-Eco-Trop., 31: 1-18.

Sonké B, Taedoumg H, Robbrecht E. 2012. A reconsideration of the Lower Guinean species of **Sericanthe** (Rubiaceae, Coffeeae), with four new species from Cameroon and Gabon. Bot. J. Linn Soc., 169(3): 530-554.

Troupin G. 1985. Flore $d u$ Rwanda. Spermatophytes (vol 3). Agence de Coopération Culturelle et Technique. Musée Royal de l'Afrique Centrale: Tervuren, Belgique.

Vancutsem C, Pekel J-F, Evrard C, Malaisse F, Defourny P. 2006. Carte de l'Occupation du Sol de la République Démocratique $d u$ Congo. Presses Universitaires de Louvain : Belgique.

White F. 1993. The chorological classification of history, methods and application. Bull. Jard. Bot. Nat. Belg., 62(2/3): 225-282.

Yamagiwa J, Basabose AK., Kaleme K, Yumoto T. 2005. Diet of grauer's gorilla in the montane forest of Kahuzi, DRC. Inte. J. Primat., 26(6): 1345-1373. 